\title{
Chinese Herbal Formulation LC09
}

National Cancer Institute

\section{Source}

National Cancer Institute. Chinese Herbal Formulation LCO9. NCI Thesaurus. Code C128893.

A traditional Chinese medicine (TCM) decoction containing Herba Epimedii that can be used for chemotherapy-induced hand-foot syndrome (HFS; Palmar-Plantar Erythrodysesthesia; PPE). Soaking of the affected hands and feet with TCM Formula LC09 may reduce the severity of HFS and may decrease HFS-associated pain. 